\title{
Ações de enfermeiros no manejo do paciente em tratamento quimioterápico: Revisão integrativa da literatura
}

\author{
Nurses' actions in the management of patients undergoing chemotherapy: An integrative literature \\ review
}

Acciones de las enfermeras en el manejo de pacientes sometidos a quimioterapia: Una revisión integradora de la literatura

Recebido: 05/05/2021 | Revisado: 13/05/2021 | Aceito: 17/05/2021 | Publicado: 04/06/2021

Sara Soares Ferreira da Silva

ORCID: https://orcid.org/0000-0001-8754-4438

Universidade Federal do Estado do Rio de Janeiro, Brasil

E-mail: sarasferreira@edu.unirio.br

Clara Beatriz Teixeira Lima Cavalcante

ORCID: https://orcid.org/0000-0003-4798-7343

Universidade Federal do Estado do Rio de Janeiro, Brasil E-mail: Clara.beatriz@edu.unirio.br

Mahanny de Souza Anizio

ORCID: https://orcid.org/0000-0002-8046-2883 Universidade Federal do Estado do Rio de Janeiro, Brasil E-mail: mahanny96@hotmail.com

Alexia Gabriele de Oliveira Sobreira

ORCID: https://orcid.org/0000-0002-4868-833X Universidade Federal do Estado do Rio de Janeiro, Brasil E-mail: alexia.sobreira@edu.unirio.br

Julia Marques de Oliveira Santos

ORCID: https://orcid.org/0000-0002-9362-5653 Universidade Federal do Estado do Rio de Janeiro, Brasil E-mail: itsjuliamm2@gmail.com

Ana Cristina Silva Pinto

ORCID: https://orcid.org/0000-0002-5608-2418 Universidade Federal do Estado do Rio de Janeiro, Brasil

E-mail: ana.3105@hotmail.com

Sônia Regina de Souza

ORCID: https://orcid.org/0000-0001-7981-0038 Universidade Federal do Estado do Rio de Janeiro, Brasil E-mail: sonia.souza@unirio.br

Laísa Figueiredo Ferreira Lós de Alcântara ORCID: https://orcid.org/0000-0002-2435-7616 Universidade Federal do Estado do Rio de Janeiro, Brasil E-mail: dra.laisa@gmail.com

Raquel de Souza Ramos

ORCID: https://orcid.org/0000-0003-1939-7864 Instituto Nacional do Câncer, Brasil E-mail: kakelramos@gmail.com

Danielle Galdino de Paula

ORCID: https://orcid.org/0000-0002-0103-6828 Universidade Federal do Estado do Rio de Janeiro, Brasil E-mail: danielle.paula@unirio.br

\section{Resumo}

Objetivo: Compreender as ações de Enfermeiros no manejo ao paciente oncológico em tratamento quimioterápico e seus impactos. Metodologia: Trata-se de estudo de revisão integrativa, realizada em seis etapas, com abordagem quantitativa. Foram elencados estudos voltados às ações de Enfermeiros no manejo de pacientes em tratamento quimioterápico, no contexto brasileiro. O recorte temporal abrangeu estudos do período de 2016 a 2020, nos idiomas inglês, português e espanhol. Resultados: Foram selecionados 17 estudos, os quais apresentaram os seguintes grupos temáticos: 1) Ações realizadas por Enfermeiros no manejo ao paciente em tratamento quimioterápico e; 2) Impactos gerados pelas referidas ações. No que se refere às ações realizadas, foi possível identificar que essas ocorrem para além da assistência, sendo evidenciadas ações em gestão e em educação em saúde, inclusive na forma de produções 
tecnológicas que foram amplamente demonstradas nos estudos, com ênfase para elaboração de manuais, protocolos e materiais didáticos. No que tange aos impactos gerados pelas ações dos Enfermeiros, foram elementos de destaque nos estudos: promoção do acesso à educação em saúde; alívio da carga de estresse do paciente e a prestação do cuidado de forma individualizada. Conclusão: Foi possível evidenciar as diversas ações dos Enfermeiros em todos os momentos do processo quimioterápico, indo além do âmbito assistencial, demonstrando a importância de tal categoria profissional no referido contexto. Ademais, foi evidenciado que enfermeiros se utilizam rotineiramente da elaboração de novas tecnologias para melhoria de processos profissionais, gerando impactos positivos e contribuindo diretamente para o aprimoramento do manejo do paciente oncológico.

Palavras-chave: Enfermagem oncológica; Cuidados de enfermagem; Quimioterapia; Melhoria de qualidade.

\begin{abstract}
Objective: To understand the actions of Nurses in the management of cancer patients undergoing chemotherapy and their impacts. Methodology: This is an integrative review study, carried out in six stages, with a quantitative approach. Studies on the actions of Nurses in the management of patients undergoing chemotherapy treatment were listed in the Brazilian context. The time frame covered studies from the period 2016 to 2020, in English, Portuguese and Spanish. Results: 17 studies were selected, which presented the following thematic groups: 1) Actions performed by Nurses in the management of patients undergoing chemotherapy and; 2) Impacts generated by the referred actions. With regard to the actions carried out, it was possible to identify that they occur beyond assistance, with actions in health management and education being evidenced, including in the form of technological productions that were widely demonstrated in studies, with emphasis on the preparation of manuals, protocols and teaching materials. Regarding the impacts generated by the actions of Nurses, the following elements were highlighted in the studies: promoting access to health education; relieving the patient's stress load and providing care individually. Conclusion: It was possible to highlight the various actions of nurses at all times in the chemotherapy process, going beyond the scope of care, demonstrating the importance of such a professional category in that context. In addition, it was evidenced that nurses routinely use the development of new technologies to improve professional processes, generating positive impacts and contributing directly to improving the management of cancer patients.
\end{abstract}

Keywords: Oncology nursing; Nursing care; Drug therapy; Quality improvement.

\title{
Resumen
}

Objetivo: Comprender las acciones de las enfermeras en el manejo de pacientes oncológicos sometidos a quimioterapia y sus impactos. Metodología: Se trata de un estudio de revisión integradora, realizado en seis etapas, con un enfoque cuantitativo. Se enumeraron los estudios sobre las acciones de las enfermeras en el manejo de los pacientes en tratamiento de quimioterapia en el contexto brasileño. El período abarcó los estudios del período 2016 a 2020, en inglés, portugués y español. Resultados: Se seleccionaron 17 estudios, los cuales presentaron los siguientes grupos temáticos: 1) Acciones realizadas por Enfermeros en el manejo de pacientes sometidos a quimioterapia y; 2) Impactos generados por las acciones referidas. En cuanto a las acciones realizadas, se pudo identificar que ocurren más allá de la asistencia, evidenciando acciones en gestión y educación en salud, incluso en la forma de producciones tecnológicas que fueron ampliamente demostradas en estudios, con énfasis en la elaboración de manuales, protocolos y material didáctico. En cuanto a los impactos generados por las acciones de Enfermería, se destacaron los siguientes elementos en los estudios: promover el acceso a la educación en salud; aliviar la carga de estrés del paciente y brindar atención individualmente. Conclusión: se pudo destacar las diversas acciones del Enfermero en todo momento durante el proceso de quimioterapia, yendo más allá del ámbito asistencial, demostrando la importancia de tal categoría profesional en ese contexto. Además, se evidenció que los enfermeros utilizan de manera rutinaria el desarrollo de nuevas tecnologías para mejorar los procesos profesionales, generando impactos positivos y contribuyendo directamente a mejorar el manejo de los pacientes oncológicos.

Palabras clave: Enfermería oncológica; Atención de enfermería; Quimioterapia; Mejora de calidad.

\section{Introdução}

O câncer consiste em um conjunto de mais de 100 tipos diferentes de doenças que compartilham entre si a característica do crescimento desordenado das células. Sua origem data de, no mínimo, mais de 3 mil anos antes de Cristo, contudo até os dias de hoje proporciona diversas questões e desafios a serem enfrentados (Instituto Nacional de Câncer José Alencar Gomes da Silva [INCA], 2020).

Atualmente, o câncer representa o maior problema de saúde pública do mundo e está entre as principais causas de morte prematura, isto é, antes dos 70 anos de idade (Bryan et al., 2018). De acordo com estimativas epidemiológicas para o Brasil, para cada ano do triênio 2020-2022, ocorrerão 625 mil novos casos de câncer, sendo o câncer de pele não-melanoma o mais incidente, seguido pelo câncer de mama, próstata e intestino (INCA, 2020). 
Dentre as possibilidades de intervenções terapêuticas para o câncer se insere a quimioterapia que consiste em um tratamento sistêmico no qual se utiliza fármacos, denominados quimioterápicos ou antineoplásicos, administrados em intervalos regulares de acordo com a individualidade requerida pela situação de cada paciente (INCA, 2020; Jemal et al., 2019).

A quimioterapia pode ser empregada para o alcance de diversos resultados, sendo eles: tornar os tumores ressecáveis ou melhorar o prognóstico do indivíduo acometido (quimioterapia prévia, neoadjuvante ou citorredutora); promover profilaxia após realização de cirurgia para ressecção de tumor (profilática ou adjuvante); proporcionar a cura (quimioterapia curativa); aumentar sobrevida (quimioterapia para controle temporário da doença) e paliar sinais e sintomas gerados por tumores, porém sem a intenção de aumentar a sobrevida (quimioterapia paliativa) (INCA, 2020).

A literatura aponta que o ambiente de realização da referida modalidade de tratamento pode proporcionar ao paciente pensamentos e sentimentos negativos, com grande relação com a morte e vulnerabilidades individuais, sendo tal circunstância agravada devido aos próprios efeitos gerados pelos antineoplásicos e seu processo de administração (Pinto, Guimarães \& Lanza, 2017).

Neste contexto, na equipe multiprofissional da assistência ao paciente oncológico, destaca-se a enfermagem que possui protagonismo nas unidades de infusão de quimioterapia, com ações nos processos de promoção da educação em saúde, pré-quimioterapia, infusão dos fármacos, manejo dos sintomas adversos e sua prevenção (Oliveira et al., 2019; Bertolazzi \& Perroca, 2020). Contudo, tais ações não se restringem ao aspecto assistencial, de forma que se estendem às áreas gerenciais, educacionais e relacionadas à pesquisa, estando presentes no cotidiano da Enfermagem.

Portanto, diante de tamanha relevância da temática, o presente artigo tem como objetivo compreender as ações de Enfermeiros no manejo ao paciente oncológico em tratamento quimioterápico e seus impactos.

\section{Metodologia}

Trata-se de uma revisão do tipo integrativa realizada em seis etapas. Esta modalidade de revisão possibilita o entendimento de determinado tema e o preenchimento de lacunas do arcabouço científico a partir de estudos selecionados de forma metódica (Mendes, Silveira \& Galvão et al., 2008).

Na primeira etapa foi definido o tema e, através da estratégia PICO, a questão norteadora. A saber, PICO se refere a um acrônimo que é definido por: Patient/Problem (paciente ou problema), Intervention (intervenção ou exposição), Comparision (intervenção de comparação ou exposição) e Outcomes (resultados) (Eriksen \& Frandsen, 2018).

Desta forma, foram definidos para o presente estudo P: Enfermagem Oncológica; I: Cuidados de Enfermagem; C: Tratamento quimioterápico e O: Melhoria de qualidade, sendo a questão norteadora definida como: "O que a literatura científica aponta sobre as ações de Enfermeiros e seus impactos no manejo ao paciente oncológico em tratamento quimioterápico?" e, ainda, foram definidas as seguintes hipóteses: 1) Existem diversas ações realizadas por Enfermeiros no manejo do paciente oncológico em tratamento quimioterápico; 2) Existem determinadas ações dos Enfermeiros, no contexto quimioterápico, que se destacam na literatura; 3) As ações realizadas por Enfermeiro direcionadas ao manejo dos pacientes oncológicos, no contexto da quimioterapia, geram impactos positivos e melhoria na qualidade da assistência.

A busca foi realizada no período de setembro a outubro de 2020 por dois juízes, de forma independente, com os descritores definidos pela estratégia PICO, oriundos do portal de Descritores de Ciências da Saúde (Decs/Mesh), utilizados de forma isolada e combinada através do operador booleano "AND”. Foram utilizadas as seguintes bases de dados: Biblioteca de Enfermagem (BDENF), Literatura Latino Americana e do Caribe em Ciências da Saúde (LILACS) e Medical Literature Analysis and Retrieval System Online (MEDLINE), através da Biblioteca Virtual em Saúde (BVS), Eletronic Library Online (Scielo), através do portal Web of Science. Para acessá-las foi utilizado o Portal de Periódicos da Capes e o serviço da 
Comunidade Acadêmica Federada (Capes Cafe). O software Mendeley® foi utilizado como ferramenta de gerenciamento de referências.

$\mathrm{Na}$ segunda etapa foram definidos os critérios de inclusão e exclusão, sendo inclusos os estudos de inteiro teor, do período de 2016 a 2020, em inglês, português ou espanhol, que abordavam as ações de Enfermeiros em relação ao manejo de pacientes oncológicos em tratamento quimioterápico no contexto brasileiro. Foram excluídos estudos que abordavam categorias da enfermagem que não fossem Enfermeiros, demais categorias profissionais da área da saúde, estudos que retratavam realidades de outros países que não o Brasil, artigos de revisão da literatura, teses, dissertações, monografias, editoriais, resumos e publicações de anais de eventos. A Figura 1 demonstra o processo de seleção dos artigos.

Figura 1. Fluxograma do processo de identificação, seleção, eleição e inclusão dos artigos, Rio de Janeiro, RJ, Brasil, 2021.

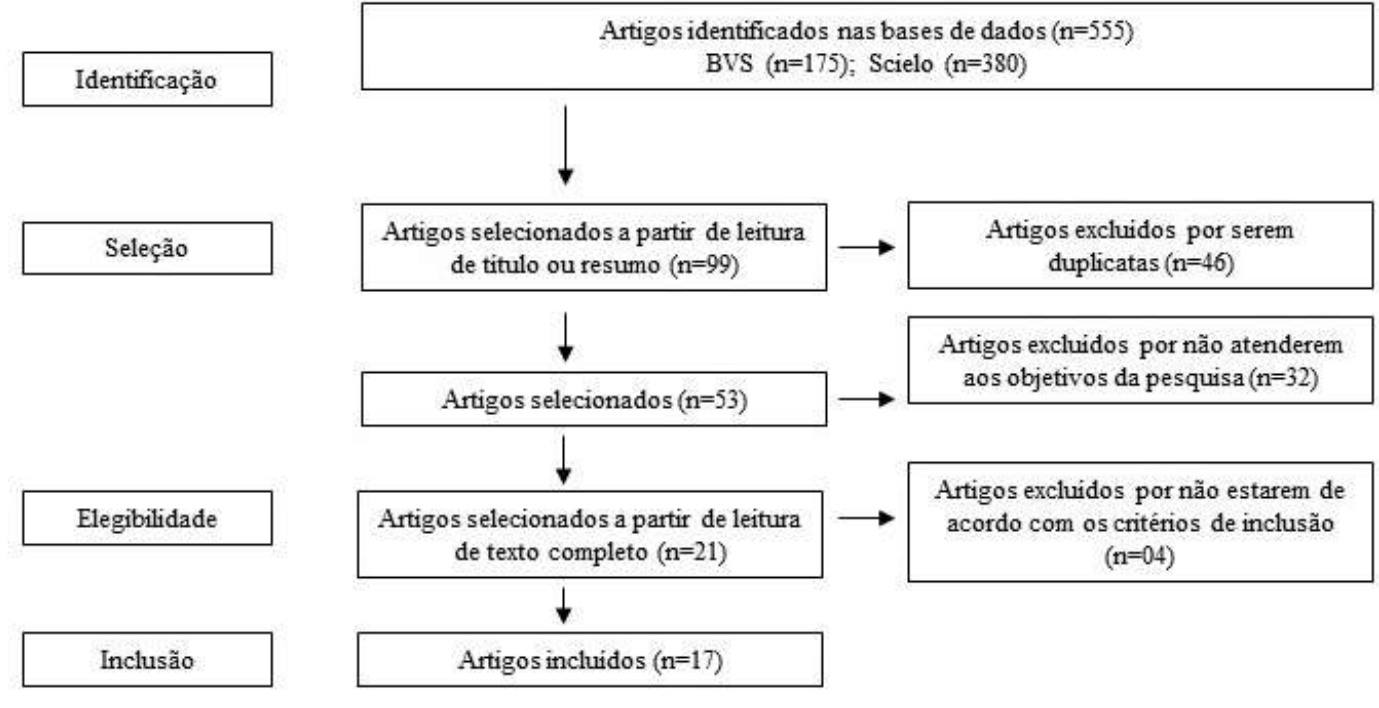

Fonte: Dados da Pesquisa (2021).

$\mathrm{Na}$ terceira etapa foram utilizados instrumentos de coleta de dados elaborados pelos autores a fim de construir um banco de dados com as principais informações dos artigos elencados, sendo descritas informações como título do artigo, ano de publicação, periódico de publicação e objetivo do estudo (Quadro 1). Além disso, dados sobre o desenho metodológico dos estudos, população do estudo, intervenções e impactos da assistência do Enfermeiro ao paciente oncológico em tratamento quimioterápico foram reunidos para posterior análise.

Na quarta etapa foi avaliado o nível de evidência dos estudos por dois juízes de forma independente ao qual, ao haver discordância, um terceiro juiz realizava a avaliação e o desempate. O nível de evidência foi definido da seguinte forma: Nível I: metanálise/revisão sistemática; II: ensaio clínico controlado randomizado; III: ensaio clínico controlado sem randomização, IV: estudo caso-controle ou coorte bem delineados; V: revisão sistemática de estudos qualitativos e descritivos, VI: estudos descritivos ou qualitativos e VII: opinião de autoridades ou relato de especialistas (Melnyk \& Fineout-Overholt, 2011). Os resultados deste processo são demonstrados no Quadro 1.

$\mathrm{Na}$ quinta etapa foi realizada a interpretação dos resultados através de análise quantitativa e na sexta etapa foi realizada a apresentação dos resultados. 
Quadro 1. Síntese dos artigos elencados conforme título, periódico de publicação, ano de publicação e nível de evidência, Rio de Janeiro, RJ, Brasil, 2021.

\begin{tabular}{|c|c|c|c|}
\hline Título do artigo & $\begin{array}{l}\text { Revista e ano } \\
\text { de publicação }\end{array}$ & Objetivo do estudo & $\begin{array}{c}\text { Nível de } \\
\text { Evidência }\end{array}$ \\
\hline $\begin{array}{l}\text { A1. Processo de enfermagem para paciente } \\
\text { com fobia de agulha: estudo de caso } \\
\text { (Mendonça, Pereira, Magnago, Silva \&Martins, } \\
\text { 2020) }\end{array}$ & $\begin{array}{l}\text { Revista } \\
\text { Brasileira de } \\
\text { Enfermagem } \\
2020\end{array}$ & $\begin{array}{l}\text { Reportar um caso clínico de fobia de agulha que } \\
\text { culminou em parada cardiorrespiratória e descrever } \\
\text { os resultados de um plano assistencial voltado aos } \\
\text { diagnósticos de medo e ansiedade, tendo como } \\
\text { referencial o Modelo de Adaptação de Roy. }\end{array}$ & IV \\
\hline $\begin{array}{l}\text { A2. Construção e avaliação de bundle frente ao } \\
\text { extravasamento de antineoplásicos: estudo } \\
\text { metodológico } \\
\text { (Melo et al., 2020) }\end{array}$ & $\begin{array}{l}\text { Acta paul. } \\
\text { enferm. } \\
2020\end{array}$ & $\begin{array}{l}\text { Construir e avaliar o conteúdo de um bundle de } \\
\text { prevenção e condutas frente ao extravasamento de } \\
\text { agentes antineoplásicos em pacientes oncológicos } \\
\text { adultos. }\end{array}$ & IV \\
\hline $\begin{array}{l}\text { A3. Diagnósticos de enfermagem associados à } \\
\text { qualidade de vida de mulheres com câncer de } \\
\text { mama em quimioterapia } \\
\text { (Naziazenoet al., 2020) }\end{array}$ & $\begin{array}{l}\text { J. res.: } \\
\text { fundam. care. } \\
\text { online } \\
2020\end{array}$ & $\begin{array}{l}\text { Identificar diagnósticos de enfermagem em mulheres } \\
\text { com câncer de mama em quimioterapia por meio do } \\
\text { mapeamento cruzado dos problemas de saúde com a } \\
\text { taxonomia NANDA-I. }\end{array}$ & VI \\
\hline $\begin{array}{l}\text { A4. Telenfermagem para controle de náuseas e } \\
\text { vômitos induzidos por quimioterapia: ensaio } \\
\text { clínico randomizado } \\
\text { (França et al., 2019) }\end{array}$ & $\begin{array}{l}\text { Texto \& } \\
\text { Contexto - } \\
\text { Enfermagem } \\
2019\end{array}$ & $\begin{array}{l}\text { Verificar a eficácia da telenfermagem no controle de } \\
\text { náuseas e vômitos induzidos pela quimioterapia } \\
\text { antineoplásica. }\end{array}$ & II \\
\hline $\begin{array}{l}\text { A5.Tecnologia educativa para manejo da fadiga } \\
\text { relacionada à quimioterapia antineoplásica } \\
\text { (Perdigão et al., 2019) }\end{array}$ & $\begin{array}{l}\text { Revista } \\
\text { Brasileira de } \\
\text { Enfermagem } \\
2019\end{array}$ & $\begin{array}{l}\text { Construir e validar tecnologia educativa (TE) sobre } \\
\text { fadiga e estratégias não farmacológicas para manejo } \\
\text { desse sintoma em pessoas com câncer em tratamento } \\
\text { quimioterápico antineoplásico ambulatorial. }\end{array}$ & VI \\
\hline $\begin{array}{l}\text { A6. Construção e validação de instrumento para } \\
\text { consulta de enfermagem em quimioterapia } \\
\text { ambulatorial } \\
\text { (Tolentino, Bettencourt \& Fonseca, 2019) }\end{array}$ & $\begin{array}{l}\text { Revista } \\
\text { Brasileira de } \\
\text { enfermagem } \\
2019\end{array}$ & $\begin{array}{l}\text { Construir e validar conteúdo de instrumento para } \\
\text { consulta de enfermagem em ambulatório de } \\
\text { quimioterapia de adultos. }\end{array}$ & III \\
\hline $\begin{array}{l}\text { A7. Abordagem Educativa ao Paciente } \\
\text { Oncológico: Estratégias para Orientação acerca do } \\
\text { Tratamento Quimioterápico } \\
\text { (Silva, Signor, Pilati, Dalfollo\& Oliveira, 2019) }\end{array}$ & $\begin{array}{l}\text { Revista } \\
\text { Brasileira de } \\
\text { Cancerologia } \\
2019\end{array}$ & $\begin{array}{l}\text { Propor um modelo de orientação ao paciente } \\
\text { oncológico acerca do tratamento quimioterápico, por } \\
\text { meio de um informativo impresso e da criação do } \\
\text { "diário do paciente". }\end{array}$ & II \\
\hline $\begin{array}{l}\text { A8. Fatores intervenientes para o cuidado de } \\
\text { enfermagem na alimentação da criança } \\
\text { hospitalizada em quimioterapia } \\
\text { (Sueiroet al., 2019) }\end{array}$ & $\begin{array}{l}\text { Revista de } \\
\text { Enfermagem } \\
\text { da UFSM - } \\
\text { REUFSM } \\
2019\end{array}$ & $\begin{array}{l}\text { Descrever os fatores intervenientes para o cuidado } \\
\text { de enfermagem na alimentação da criança } \\
\text { hospitalizada em quimioterapia antineoplásica. }\end{array}$ & VI \\
\hline $\begin{array}{l}\text { A9. Cuidados de enfermagem da alimentação de } \\
\text { crianças em quimioterapia: contribuições de } \\
\text { collière } \\
\text { (Sueiroet al., 2019) }\end{array}$ & $\begin{array}{l}\text { J. res.: } \\
\text { fundam. care. } \\
\text { online } \\
2019\end{array}$ & $\begin{array}{l}\text { Compreender os cuidados de enfermagem frente às } \\
\text { alterações no padrão alimentar de crianças em } \\
\text { quimioterapia antineoplásica à luz de Collière. }\end{array}$ & VI \\
\hline $\begin{array}{l}\text { A10.Diagnósticos de enfermagem em pacientes } \\
\text { oncohematológicos submetidos a tratamento } \\
\text { quimioterápico } \\
\text { (Calegari, Cordeiro, Stacciarini\& Ferreira, 2018) }\end{array}$ & $\begin{array}{l}\text { Revista de } \\
\text { Enfermagem } \\
\text { e Atenção à } \\
\text { Saúde - } \\
\text { REAS / } \\
\text { UFTM } \\
2018\end{array}$ & $\begin{array}{l}\text { Identificar os diagnósticos de Enfermagem mais } \\
\text { frequentes em pacientes com doenças } \\
\text { oncohematológicas submetidos a tratamento } \\
\text { quimioterápico antineoplásico. }\end{array}$ & II \\
\hline
\end{tabular}




\begin{tabular}{|c|c|c|c|}
\hline $\begin{array}{l}\text { A11.Contato telefônico como estratégia para a } \\
\text { promoção de conforto ao paciente submetido à } \\
\text { quimioterapia } \\
\text { (Ferreira et al., 2017) }\end{array}$ & $\begin{array}{l}\text { Rev enferm } \\
\text { UFPE on line. } \\
2017\end{array}$ & $\begin{array}{l}\text { Monitorar os efeitos adversos da quimioterapia } \\
\text { antineoplásica em pacientes submetidos a tratamento } \\
\text { ambulatorial por meio do acompanhamento } \\
\text { telefônico enquanto estratégia de provimento de } \\
\text { conforto, de acordo com os pressupostos de } \\
\text { Katherine Kolcaba. }\end{array}$ & VI \\
\hline $\begin{array}{l}\text { A12.Representações de pacientes oncológicos } \\
\text { sobre o tratamento de quimioterapia antineoplásica } \\
\text { (Cunha, Vasconcelos, Silva \& Freitas, 2017) }\end{array}$ & $\begin{array}{l}\text { J. res.: } \\
\text { fundam. care. } \\
\text { online } \\
2017\end{array}$ & $\begin{array}{l}\text { Compreender e identificar as representações de } \\
\text { pacientes oncológicos sobre o tratamento de } \\
\text { quimioterapia antineoplásica e o cuidado de } \\
\text { enfermagem. }\end{array}$ & VI \\
\hline $\begin{array}{l}\text { A13.Implementação de manuais educativos na } \\
\text { consulta de enfermagem: opinião dos pacientes } \\
\text { submetidos à quimioterapia antineoplásica } \\
\text { (Cruz et al., 2017) }\end{array}$ & $\begin{array}{l}\text { Rev enferm } \\
\text { UFPE on line } \\
2017\end{array}$ & $\begin{array}{l}\text { Conhecer a opinião dos pacientes com relação aos } \\
\text { manuais educativos implementados durante a } \\
\text { consulta de Enfermagem prévia à quimioterapia } \\
\text { antineoplásica }\end{array}$ & VI \\
\hline $\begin{array}{l}\text { A14.A gerência do cuidado de enfermagem à } \\
\text { mulher com câncer de mama em quimioterapia } \\
\text { paliativa } \\
\text { (Cirilo, Silva, Fuly \& Moreira, 2016) }\end{array}$ & $\begin{array}{l}\text { Texto } \\
\text { Contexto } \\
\text { Enferm } \\
2016\end{array}$ & $\begin{array}{l}\text { Compreender e analisar a gerência do cuidado de } \\
\text { enfermagem à mulher com câncer de mama em } \\
\text { quimioterapia paliativa }\end{array}$ & VI \\
\hline $\begin{array}{l}\text { A15.Emergência oncológica: atuação dos } \\
\text { enfermeiros no extravasamento de drogas } \\
\text { quimioterápicas antineoplásicas } \\
\text { (Souza et al., 2016) }\end{array}$ & $\begin{array}{l}\text { Escola Anna } \\
\text { Nery } \\
2016\end{array}$ & $\begin{array}{l}\text { Investigar a atuação dos enfermeiros no } \\
\text { extravasamento de quimioterápicos antineoplásicos. }\end{array}$ & VI \\
\hline $\begin{array}{l}\text { A16.Prevenção e tratamento da mucosite em } \\
\text { ambulatório de oncologia: uma construção coletiva } \\
\text { (Lopes et al., 2016) }\end{array}$ & $\begin{array}{l}\text { Texto } \\
\text { Contexto } \\
\text { Enferm } \\
2016\end{array}$ & $\begin{array}{l}\text { Elaborar um protocolo assistencial de enfermagem } \\
\text { para prevenção e tratamento da mucosite induzida } \\
\text { por quimioterapia em um ambulatório de um Centro } \\
\text { de Alta Complexidade em Oncologia. }\end{array}$ & VI \\
\hline $\begin{array}{l}\text { A17.Autocuidado e quimioterapia oral domiciliar: } \\
\text { avaliação das práticas educativas dos enfermeiros } \\
\text { sob a perspectiva de pacientes } \\
\text { (Mesquita \& Silva, 2016) }\end{array}$ & $\begin{array}{l}\text { Rev. Bras. } \\
\text { Cancerol. } \\
2016\end{array}$ & $\begin{array}{l}\text { Avaliar, na perspectiva dos pacientes, convergências } \\
\text { e divergências entre orientações para o autocuidado, } \\
\text { compreensão quanto às orientações e sua aplicação. }\end{array}$ & VI \\
\hline
\end{tabular}

Fonte: Dados da pesquisa (2021).

\section{Resultados e Discussão}

Foram selecionados 17 artigos, sendo 1 artigo (5,8\%) apenas na língua inglesa, 3 artigos (17,6\%) apenas na língua portuguesa, 12 artigos (70,5\%) na língua portuguesa e inglesa e 1 artigo (5,8\%) na língua portuguesa, inglesa e espanhola. Destes, a maioria foi publicada no ano de 2019 ( $n=6,35,3 \%)$, seguido pelo ano de $2016(n=4,23,5 \%), 2017$ e $2020(n=3$, $17,6 \%)$ e 2018 com uma publicação $(n=1,5 \%)$.

Quanto a localização dos cenários de estudo no Brasil, 6 artigos tiveram como localização de cenário o Rio de Janeiro ( $\mathrm{n}=6,35,3 \%), 2$ artigos em Brasília $(\mathrm{n}=2,11,7 \%), 1$ artigo em São Paulo, Minas Gerais, Pernambuco, Sergipe e Pará $(\mathrm{n}=1$, 5,8\%), 3 artigos no Ceará $(\mathrm{n}=3,17,6 \%)$ e 1 artigo não especificou o Estado, apenas mencionou ser no sudeste brasileiro $(\mathrm{n}=1$, $5,8 \%)$.

No que se refere ao tipo de câncer abordado nos estudos, 11 artigos (64,7\%) não especificam um determinado tipo, abarcando uma ampla gama de tipos de câncer, 2 (11,7\%) são relativos ao câncer de mama, 1 artigo $(5,8 \%)$ aborda especificamente pacientes com câncer hematológico, 1 (5,8\%) o câncer colorretal, 1 (5,8\%) o câncer gástrico e colorretal e 1 $(5,8 \%)$ ao câncer de seio endodérmico.

Quanto ao recorte apresentado pelos estudos relativos a características da população amostral, 11 artigos (64,7\%) delimitaram sua população segundo a faixa etária, sendo composta por adultos, contudo não houve delimitação por sexo, 2 
artigos $(11,7 \%)$ delimitaram sua população amostral ao sexo feminino, 2 artigos $(11,7 \%)$ delimitaram a faixa etária infantil e 2 artigos $(11,7 \%)$ não definiram recortes relacionados a faixa etária e sexo.

No que tange às especificidades dos tratamentos quimioterápicos empregados nos estudos, 13 artigos (76,4\%) não forneceram informações específicas sobre a modalidade de quimioterapia empregada, 1 artigo $(5,8 \%)$ fez menção a quimioterapia nas modalidades neoadjuvante, adjuvante e curativa, 1 artigo $(5,8 \%)$ menciona quimioterapia paliativa. Ainda, 1 artigo $(5,8 \%)$ salienta a quimioterapia empregada em sua população do estudo como possuindo relevante potencial emetogênico e 1 artigo (5,8\%) cita a quimioterapia oral, contudo não apresentou maiores especificações.

Quanto ao nível de evidência, notou-se a frequência de 3 artigos no nível II: ensaio clínico controlado randomizado ( $\mathrm{n}=3,17,6 \%), 1$ artigo no nível III: ensaio clínico controlado sem randomização (n=1, 5,8\%), 2 artigos no nível IV: estudo caso-controle ou coorte bem delineados $(n=2,11,9 \%)$ e 11 artigos no nível VI: estudos descritivos ou qualitativos ( $\mathrm{n}=11$, $64,7 \%)$.

A partir dos estudos selecionados, duas categorias temáticas foram definidas: (1) Ações realizadas por Enfermeiros no manejo ao paciente em tratamento quimioterápico e (2) Impactos gerados pelas ações de Enfermeiros direcionadas ao manejo de pacientes em tratamento quimioterápico.

\section{Ações realizadas por Enfermeiros no manejo ao paciente em tratamento quimioterápico}

Foi possível identificar que as ações de enfermagem se deram em distintos âmbitos, sendo eles: (1) Assistência ao paciente oncológico em quimioterapia; (2) Ações em gestão e; (3) Ações em educação em saúde, sendo elas esmiuçadas a seguir.

Quanto às ações de enfermagem relacionadas à assistência ao paciente em quimioterapia, descritas nos estudos, seis categorias distintas foram identificadas e demonstradas na Tabela 1. As classificações foram realizadas conforme o que os estudos apontaram como ações realizadas por Enfermeiros, sendo importante salientar que diversos artigos contaram com mais de uma das categorias identificadas. 
Tabela 1. Ações realizadas por Enfermeiros na assistência aos pacientes em tratamento quimioterápico. Rio de Janeiro, RJ, Brasil, 2021.

\begin{tabular}{llll}
\hline Ações de Enfermeiros & N & \% \\
\hline Consulta de Enfermagem & 06 & 35,2 \\
\hline
\end{tabular}

Artigo 6: Consulta de enfermagem em ambulatório de quimioterapia de adultos

Artigo 4, 11 e 13: Realização de teleconsulta de enfermagem, através de contato telefônico, para orientações

Artigo 13: Consulta de enfermagem pré-quimioterapia

Artigo 14: Consulta realizada na admissão em ambulatório quimioterápico

Orientações em saúde

Artigo 8: Orientações voltadas à alimentação da criança hospitalizada em quimioterapia

Artigo 12: Orientações quanto ao autocuidado e esclarecimento de dúvidas relacionadas ao processo de tratamento

Artigo 14: Orientação sobre as questões relativas à quimioterapia paliativa

Artigo 17: Orientações em saúde voltadas ao autocuidado durante quimioterapia oral domiciliar

Artigo 11: Realização de consulta de Enfermagem com orientações verbais e utilizando manual de orientações, elaborado pelos Enfermeiros, como instrumento auxiliador

Artigo 1: Identificação de intercorrências relacionada à fobia de agulhas no momento da administração da quimioterapia

Artigo 5: Manejo de sintomas adversos causados pela quimioterapia através de material educativo elaborado por enfermeiros

Artigo 9: Controle farmacológico dos sintomas relacionados aos efeitos colaterais

Artigo 4 e 11: Realização de teleconsulta de enfermagem para monitorização de efeitos adversos da quimioterapia

Apoio emocional

Artigo 8: Encorajamento da participação familiar no cuidado alimentar da criança com câncer; Oferta de apoio para enfrentamento das capacidades alteradas pela doença

Artigo 11: Orientação quanto à aspectos relacionados à autoestima e autoconhecimento;

Oferta de apoio emocional

Artigo 12: Desenvolvimento afetivo e atenção para com o paciente; Orientações quanto ao autocuidado

$$
\text { Administração de medicamentos }
$$

Artigo 9: Controle farmacológico dos sintomas relacionados aos efeitos colaterais da QT e para o controle da dor

Artigo 12: Administração de quimioterápicos

Artigo 15: Manejo relacionado ao extravasamento de antineoplásicos

Promoção da alimentação adequada

02

11,8

Artigo 8: Identificação de ações facilitadoras para promoção da alimentação adequada de crianças com câncer

Artigo 9: Elaboração de estratégias para o cuidado de enfermagem frente às alterações no padrão alimentar de crianças em quimioterapia antineoplásica 
Conforme explicitado na Tabela 1, determinadas ações se destacaram dentre as demais, como a realização de consulta de enfermagem, ganhando destaque a modalidade de teleconsulta. A referida abordagem é apontada por estudos como capaz de promover o conforto, como apontado em relação ao alívio de náuseas e vômitos pós-quimioterapia, e vínculo entre paciente em tratamento antineoplásico e equipe de saúde que o assiste, oportunizando o reforço de orientações e identificação de fragilidades e potencialidades de forma otimizada (França et al., 2019; Ferreira et al., 2017). Desta forma, foi evidenciado que o monitoramento remoto revelou-se como uma medida complementar às ações dos Enfermeiros e ao processo de tratamento, gerando impactos positivos aos pacientes oncológicos.

A categoria Orientações em saúde evidenciou que os Enfermeiros atuam diretamente na elucidação de dúvidas e preparo do paciente quanto ao processo de tratamento quimioterápico sendo, inclusive, fundamental para estimular a adesão à terapêutica, assim como seu adequado acompanhamento (Sueiro et al., 2019; Cunha, Vasconcelos, Silva \& Freitas, 2017; Cirilo, Silva, Fuly \& Moreira, 2016; Mesquita \& Silva, 2016; Ferreira et al., 2017).

Ainda, foi possível identificar a ação do Enfermeiro na administração de antineoplásicos, sendo evidenciada sua importância no manejo de efeitos adversos e intercorrências no momento de infusão dos antineoplásicos, assim como em sua posterioridade (Sueiro et al., 2019; Cunha, Vasconcelos, Silva \& Freitas, 2017; Souza et al., 2016).

Acerca da categoria Apoio emocional, foi possível identificar a necessidade do desenvolvimento pelo Enfermeiro de um processo de cuidado no qual sejam considerados fatores socioculturais para promoção de melhoria do enfrentamento pelo paciente de sua nova realidade, desse modo, faz-se necessária a criação de estratégias específicas neste contexto, visando ampliar o conforto do paciente oncológico. Nos estudos que trouxeram tal perspectiva, a Enfermagem mostrou sua contribuição através do empoderamento do paciente e seu acolhimento de forma individual (Sueiro et al., 2019; Ferreira et al., 2017; Cunha, Vasconcelos, Silva \& Freitas, 2017).

Quanto à promoção da alimentação adequada, foi evidenciado que a população de maior destaque neste contexto é a população infantil. Nesta conjuntura, a enfermagem foi evidenciada como importante mediadora entre os profissionais da equipe multidisciplinar, a criança e os cuidadores, além de propiciar esclarecimentos que facilitam a compreensão por parte dos familiares quanto à situação alimentar das crianças. Tal medida é de suma importância visto que a integração dos cuidadores no processo de tratamento da criança é capaz de proporcionar maior apoio e segurança a ela (Sueiro et al., 2019, 2019).

No que se refere às ações em gestão e em educação em saúde, foi possível perceber que estas se fizeram presente na forma de produções tecnológicas no espaço de assistência do Enfermeiro, sendo possível identificar o protagonismo da enfermagem em processos inovadores nos diferentes cenários de atuação do contexto da quimioterapia.

Diversos produtos tecnológicos foram apontados nos estudos selecionados, sendo eles elaborados conforme demandas advindas de seus cenários de prática. Tais produtos são elucidados e classificados no Quadro 2 conforme a área correspondente de ação do enfermeiro, categoria, complexidade, teor inovativo e impacto, sendo empregado como referencial teórico o relatório do grupo de trabalho elaborado pela Coordenação de Aperfeiçoamento de Pessoal de Nível Superior (CAPES) do ano de 2019 (CAPES, 2019). 
Quadro 2. Produções técnicas/tecnológicas elaboradas por enfermeiros nos estudos selecionados, conforme critérios da CAPES (2019), Rio de Janeiro, RJ, Brasil, 2021.

\begin{tabular}{|c|c|c|c|c|}
\hline $\begin{array}{l}\text { Produto } \\
\text { Técnico/ } \\
\text { Tecnológico }\end{array}$ & Artigo & $\begin{array}{l}\text { Área de } \\
\text { ação do } \\
\text { Enfermeiro }\end{array}$ & $\begin{array}{l}\text { Categoria do } \\
\text { produto }^{1}\end{array}$ & Classificação da Produção Técnica/Tecnológica ${ }^{1}$ \\
\hline \multirow{4}{*}{$\begin{array}{l}\text { Processo de } \\
\text { enfermagem } \\
\text { voltado a } \\
\text { paciente com } \\
\text { fobia de agulha }\end{array}$} & \multirow{4}{*}{$\begin{array}{l}\text { Processo de } \\
\text { enfermagem para } \\
\text { paciente com fobia de } \\
\text { agulha: estudo de caso } \\
\text { (Mendonça, Pereira, } \\
\text { Magnago, Silva \& } \\
\text { Martins, 2019) }\end{array}$} & \multirow[t]{4}{*}{ Gestão } & \multirow{4}{*}{$\begin{array}{l}\text { Processo / } \\
\text { Tecnologia e } \\
\text { Produto / Material } \\
\text { não patenteáveis }\end{array}$} & Complexidade: alta complexidade \\
\hline & & & & $\begin{array}{l}\text { Aplicabilidade: necessita da aplicação de instrumentos, } \\
\text { já validados, voltados à avaliação do estado de } \\
\text { ansiedade/depressão e de intervenções aplicadas pelo } \\
\text { enfermeiro, sendo, portanto, de simples aplicação no } \\
\text { cotidiano }\end{array}$ \\
\hline & & & & $\begin{array}{l}\text { Impacto: planejamento de proposta assistencial a } \\
\text { paciente com fobia de agulha e estabelecimento de } \\
\text { instrumento significativo metodológico que } \\
\text { proporcionou assistência de enfermagem efetiva e } \\
\text { direcionada ao controle de estímulos focais que } \\
\text { colocam a vida do cliente em risco }\end{array}$ \\
\hline & & & & Teor de Inovação: médio teor inovativo \\
\hline \multirow{4}{*}{$\begin{array}{l}\text { Bundle voltado } \\
\text { ao } \\
\text { extravasamento } \\
\text { de } \\
\text { antineoplásicos }\end{array}$} & \multirow{4}{*}{$\begin{array}{l}\text { Construção e } \\
\text { avaliação de bundle } \\
\text { frente ao } \\
\text { extravasamento de } \\
\text { antineoplásicos: } \\
\text { estudo metodológico } \\
\text { (Melo et al., 2019) }\end{array}$} & \multirow[t]{4}{*}{ Gestão } & \multirow{4}{*}{$\begin{array}{l}\text { Manual/ } \\
\text { Protocolo }\end{array}$} & Complexidade: alta complexidade \\
\hline & & & & $\begin{array}{l}\text { Aplicabilidade: requer adesão por parte dos } \\
\text { enfermeiros assistenciais e apoio institucional para sua } \\
\text { adequada implementação, no mais, não apresenta } \\
\text { fatores que dificultem sua efetivação }\end{array}$ \\
\hline & & & & $\begin{array}{l}\text { Impacto: o objeto do estudo não foi direcionado a } \\
\text { avaliação do impacto, contudo, o estudo demonstra } \\
\text { grande potencial de impacto por permitir a } \\
\text { sistematização da assistência frente ao extravasamento } \\
\text { de antineoplásicos, tanto na prevenção quanto no seu } \\
\text { manejo de forma a beneficiar o paciente e a instituição }\end{array}$ \\
\hline & & & & Teor de Inovação: médio teor inovativo \\
\hline \multirow{4}{*}{$\begin{array}{l}\text { Definição de } \\
\text { diagnósticos de } \\
\text { enfermagem } \\
\text { voltados a } \\
\text { qualidade de vida } \\
\text { de mulheres com } \\
\text { câncer de mama } \\
\text { em quimioterapia }\end{array}$} & \multirow{4}{*}{$\begin{array}{l}\text { Diagnósticos de } \\
\text { enfermagem } \\
\text { associados à qualidade } \\
\text { de vida de mulheres } \\
\text { com câncer de mama } \\
\text { em quimioterapia } \\
\text { (Naziazeno et al., } \\
\text { 2020) }\end{array}$} & \multirow[t]{4}{*}{ Gestão } & \multirow{4}{*}{$\begin{array}{l}\text { Processo / } \\
\text { Tecnologia e } \\
\text { Produto / Material } \\
\text { não patenteáveis }\end{array}$} & Complexidade: alta complexidade \\
\hline & & & & $\begin{array}{l}\text { Aplicabilidade: produto de fácil aplicação por não } \\
\text { requerer grandes recursos e alterações do ambiente de } \\
\text { atuação, contudo pode requerer alterações de padrões } \\
\text { da atuação da enfermagem }\end{array}$ \\
\hline & & & & $\begin{array}{l}\text { Impacto: identificação dos problemas de saúde mais } \\
\text { frequentes, suscetíveis a intervenções de enfermagem, } \\
\text { que afetam as mulheres com câncer de mama em } \\
\text { quimioterapia, assim como, promove o planejamento } \\
\text { da assistência de enfermagem a mulheres com câncer } \\
\text { de mama em quimioterapia, por padronizar uma } \\
\text { linguagem diagnóstica específica para este grupo e } \\
\text { fornece subsídios ao processo de enfermagem }\end{array}$ \\
\hline & & & & Teor de Inovação: baixo teor inovativo \\
\hline
\end{tabular}




\begin{tabular}{|c|c|c|c|c|}
\hline \multirow{4}{*}{$\begin{array}{l}\text { Telenfermagem } \\
\text { para controle de } \\
\text { náuseas e } \\
\text { vômitos } \\
\text { induzidos por } \\
\text { quimioterapia }\end{array}$} & \multirow{4}{*}{$\begin{array}{l}\text { Telenfermagem para } \\
\text { controle de náuseas e } \\
\text { vômitos induzidos por } \\
\text { quimioterapia: ensaio } \\
\text { clínico randomizado } \\
\text { (França et al., 2019) }\end{array}$} & \multirow{4}{*}{$\begin{array}{l}\text { Educação em } \\
\text { saúde }\end{array}$} & \multirow{4}{*}{$\begin{array}{l}\text { Processo / } \\
\text { Tecnologia e } \\
\text { Produto / Material } \\
\text { não patenteáveis }\end{array}$} & Complexidade: média complexidade \\
\hline & & & & $\begin{array}{l}\text { Aplicabilidade: necessita de (a) recursos tecnológicos } \\
\text { por parte dos profissionais e pacientes; (b) treinamento } \\
\text { dos enfermeiros e; (c) suporte organizacional, sendo, } \\
\text { portanto, tecnologia não tão facilmente aplicável }\end{array}$ \\
\hline & & & & $\begin{array}{l}\text { Impacto: demonstrou eficiência para controle dos } \\
\text { sintomas e orientações ao paciente nos momentos de } \\
\text { maior incidência de náuseas e vômitos induzidos por } \\
\text { quimioterapia }\end{array}$ \\
\hline & & & & Teor de Inovação: baixo teor inovativo \\
\hline \multirow{4}{*}{$\begin{array}{l}\text { Conhecendo e } \\
\text { lidando com a } \\
\text { fadiga }\end{array}$} & \multirow{4}{*}{$\begin{array}{l}\text { Tecnologia educativa } \\
\text { para manejo da fadiga } \\
\text { relacionada à } \\
\text { quimioterapia } \\
\text { antineoplásica } \\
\text { (Perdigão et al., 2019) }\end{array}$} & \multirow{4}{*}{$\begin{array}{l}\text { Educação em } \\
\text { saúde }\end{array}$} & \multirow[t]{4}{*}{ Material didático } & Complexidade: alta complexidade \\
\hline & & & & $\begin{array}{l}\text { Aplicabilidade: requer recursos para sua distribuição } \\
\text { podendo ser este um agente dificultador, contudo, } \\
\text { trata-se de produto de simples aplicação no cotidiano } \\
\text { profissional. A escolaridade do público-alvo pode ser } \\
\text { empecilho para seu sucesso de implementação. }\end{array}$ \\
\hline & & & & $\begin{array}{l}\text { Impacto: não foi objetivo do estudo a demonstração do } \\
\text { impacto gerado, contudo, trata-se de produto com alto } \\
\text { potencial de impacto para seu grupo-alvo }\end{array}$ \\
\hline & & & & Teor de Inovação: médio teor inovativo \\
\hline \multirow{4}{*}{$\begin{array}{l}\text { Instrumento para } \\
\text { consulta de } \\
\text { enfermagem em } \\
\text { quimioterapia } \\
\text { ambulatorial }\end{array}$} & \multirow{4}{*}{$\begin{array}{l}\text { Construção e } \\
\text { validação de } \\
\text { instrumento para } \\
\text { consulta de } \\
\text { enfermagem em } \\
\text { quimioterapia } \\
\text { ambulatorial } \\
\text { (Tolentino, } \\
\text { Bettencourt \& } \\
\text { Fonseca, 2019) }\end{array}$} & \multirow[t]{4}{*}{ Gestão } & \multirow[t]{4}{*}{ Manual/protocolo } & Complexidade: alta complexidade \\
\hline & & & & $\begin{array}{l}\text { Aplicabilidade: instrumento de fácil aplicação devido } \\
\text { ao não requerimento de recursos financeiros e } \\
\text { tecnológicos, contudo necessita da adesão enquanto } \\
\text { nova rotina na atuação do enfermeiro }\end{array}$ \\
\hline & & & & $\begin{array}{l}\text { Impacto: o objetivo do estudo não visava a atribuição } \\
\text { do impacto do instrumento, contudo, apresenta alto } \\
\text { potencial positivo no cenário assistencial }\end{array}$ \\
\hline & & & & Teor de Inovação: médio teor inovativo \\
\hline \multirow{4}{*}{$\begin{array}{l}\text { Modelo de } \\
\text { orientação ao } \\
\text { paciente } \\
\text { oncológico sobre } \\
\text { o tratamento } \\
\text { quimioterápico: } \\
\text { material } \\
\text { informativo e } \\
\text { diário do } \\
\text { paciente }\end{array}$} & \multirow{4}{*}{$\begin{array}{l}\text { Abordagem } \\
\text { Educativa ao Paciente } \\
\text { Oncológico: } \\
\text { Estratégias para } \\
\text { Orientação acerca do } \\
\text { Tratamento } \\
\text { Quimioterápico } \\
\text { (Silva, Signor, Pilati, } \\
\text { Dalfollo \& Oliveira, } \\
\text { 2019) }\end{array}$} & \multirow[t]{4}{*}{$\begin{array}{l}\text { Educação em } \\
\text { Saúde }\end{array}$} & \multirow[t]{4}{*}{ Material didático } & Complexidade: alta complexidade \\
\hline & & & & $\begin{array}{l}\text { Aplicabilidade: requer recursos para sua reprodução } \\
\text { e distribuição podendo ser este um agente } \\
\text { dificultador, além disso, a escolaridade do paciente } \\
\text { pode ser um fator emblemático no processo de } \\
\text { aplicação, porém, trata-se de produto de simples } \\
\text { aplicação no cotidiano profissional }\end{array}$ \\
\hline & & & & $\begin{array}{l}\text { Impacto: atuação efetiva do enfermeiro na } \\
\text { abordagem educativa com paciente e familiares e } \\
\text { promoção de vínculos entre enfermeiro e paciente }\end{array}$ \\
\hline & & & & Teor de Inovação: baixo teor inovativo \\
\hline \multirow{2}{*}{$\begin{array}{l}\text { Contato } \\
\text { telefônico para a } \\
\text { promoção de } \\
\text { conforto ao } \\
\text { paciente em }\end{array}$} & \multirow{2}{*}{$\begin{array}{l}\text { Implementação de } \\
\text { manuais educativos } \\
\text { na consulta de } \\
\text { enfermagem: opinião } \\
\text { dos pacientes }\end{array}$} & \multirow{2}{*}{$\begin{array}{l}\text { Educação em } \\
\text { saúde }\end{array}$} & \multirow{2}{*}{$\begin{array}{l}\text { Processo / } \\
\text { Tecnologia e } \\
\text { Produto / Material } \\
\text { não patenteáveis }\end{array}$} & Complexidade: baixa complexidade \\
\hline & & & & $\begin{array}{l}\text { Aplicabilidade: requer recursos tecnológicos por } \\
\text { parte da instituição de atuação do enfermeiro e por } \\
\text { parte do paciente }\end{array}$ \\
\hline
\end{tabular}




\begin{tabular}{|c|c|c|c|c|}
\hline \multirow[t]{2}{*}{ quimioterapia } & \multirow[t]{2}{*}{$\begin{array}{l}\text { submetidos à } \\
\text { quimioterapia } \\
\text { antineoplásica } \\
\text { (Cruz et al., 2017) }\end{array}$} & & & $\begin{array}{l}\text { Impacto: o processo proporcionou (a) estratégias de } \\
\text { educação em saúde e de avaliação do conhecimento } \\
\text { do paciente e de seu acompanhante para processo de } \\
\text { enfrentamento e; (b) incentivo para a mudança de } \\
\text { estilo de vida e comportamento }\end{array}$ \\
\hline & & & & Teor de Inovação: baixo teor inovativo \\
\hline \multirow{4}{*}{$\begin{array}{l}\text { Protocolo } \\
\text { assistencial para } \\
\text { prevenção e } \\
\text { tratamento de } \\
\text { mucosite em } \\
\text { pacientes } \\
\text { oncológicos }\end{array}$} & \multirow{4}{*}{$\begin{array}{l}\text { Prevenção e } \\
\text { tratamento da } \\
\text { mucosite em } \\
\text { ambulatório de } \\
\text { oncologia: uma } \\
\text { construção coletiva } \\
\text { (Lopes et al., 2016) }\end{array}$} & \multirow[t]{4}{*}{ Gestão } & \multirow[t]{4}{*}{ Manual/Protocolo } & Complexidade: alta complexidade \\
\hline & & & & $\begin{array}{l}\text { Aplicabilidade: o produto requer mudança no padrão } \\
\text { de assistência prestado por enfermeiros e suporte } \\
\text { institucional, contudo, não apresenta necessidade de } \\
\text { recursos adicionais e dispendiosos }\end{array}$ \\
\hline & & & & $\begin{array}{l}\text { Impacto: padronização e sistematização da } \\
\text { assistência ao paciente em tratamento quimioterápico } \\
\text { voltado a prevenção e manejo de mucosite oral }\end{array}$ \\
\hline & & & & Teor de Inovação: baixo teor inovativo \\
\hline
\end{tabular}

Legenda: ${ }^{1}$ Segundo relatório de produção técnica da CAPES (2019). Fonte: Dados da pesquisa (2021)

O Quadro 2 evidenciou que as ações em gestão foram significativas ( $\mathrm{n}=05,55,6 \%$ ), sendo distribuídas nas categorias processo/tecnologia não patenteável $(n=02 ; 40 \%)$ e manual/protocolos ( $n=03 ; 60 \%)$. Tal resultado vem de encontro com estudo de revisão sistemática acerca de produções tecnológicas realizadas por Enfermeiros, na qual foi verificado que as produções correspondentes a categoria Manual/protocolo foi de 28,57\% e Processo/tecnologia não patenteável a 17,86\% (Silva et al, 2020).

Desta forma, é possível perceber a participação de Enfermeiros na produção tecnológica voltada à melhoria de processos de gestão que, por sua vez, culminam na melhoria da assistência ao paciente, inclusive os oncológicos, através de fluxos bem definidos, maior segurança e apoio à assistência.

Ainda, foram expressivas as ações em educação em saúde ( $\mathrm{n}=04 ; 44,5 \%)$, através da produção de materiais didáticos e processo/tecnologia não patenteável $(\mathrm{n}=02 ; 50 \%)$, respectivamente. Os produtos foram voltados ao manejo dos sintomas relacionados ao processo quimioterápico, uso de recursos não farmacológicos para manejo da fadiga pós-quimioterapia e acompanhamento da vivência do paciente através de um diário.

Portanto foi evidenciado que diversas ações são realizadas por Enfermeiros no manejo do paciente oncológico em tratamento quimioterápico, sendo imprescindíveis em todas as etapas do processo.

\section{Impactos gerados pelas ações de Enfermeiros direcionadas ao manejo de pacientes em tratamento quimioterápico}

Quanto aos impactos gerados pelas ações de Enfermeiros no contexto quimioterápico, um total de 15 categorias foram delimitadas dentre as 50 observações de impactos descritas nos estudos selecionados. Tais resultados são demonstrados na Figura 2. 
Figura 2. Impactos gerados pelas ações de Enfermeiros identificados nos estudos primários, Rio de Janeiro, RJ, Brasil, 2021.

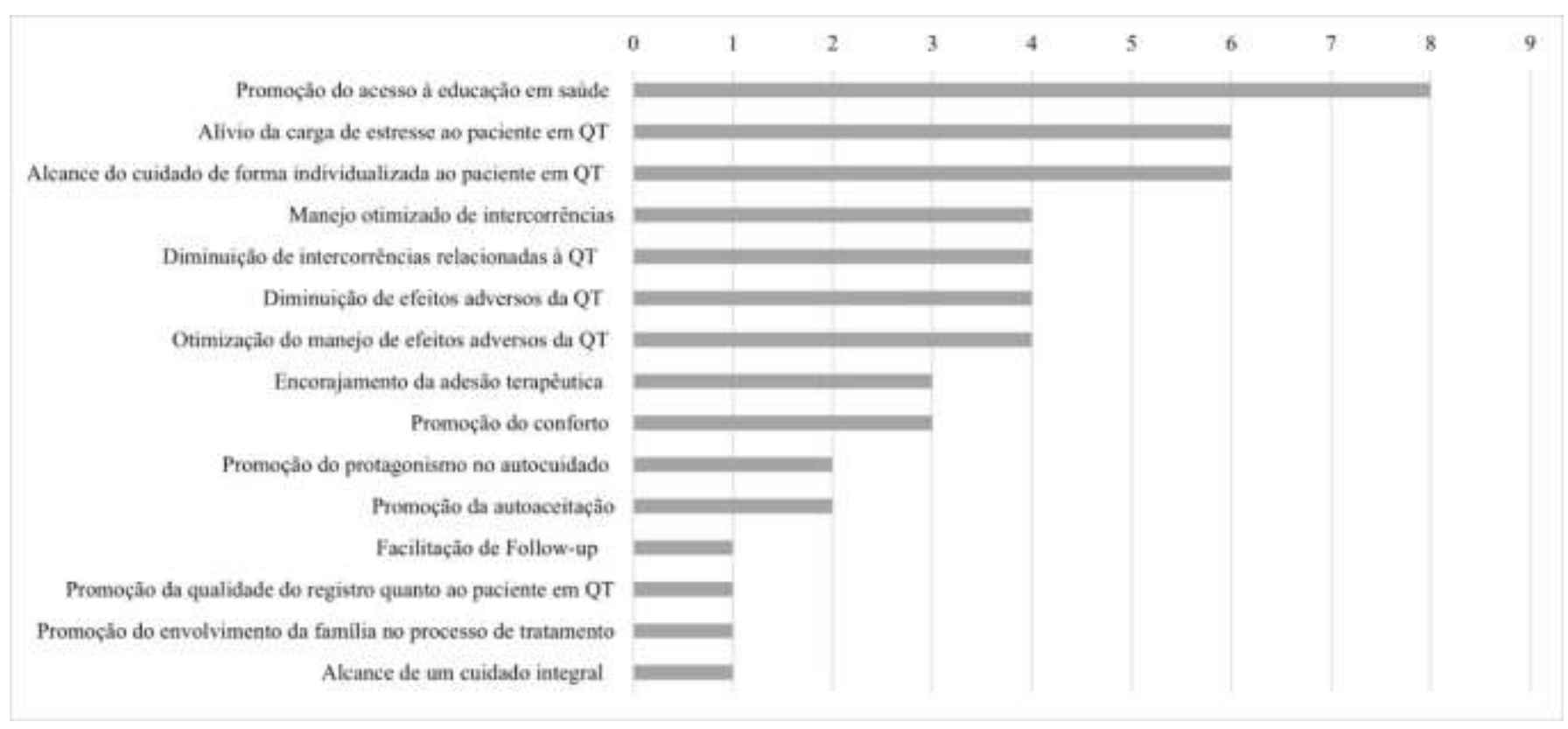

Legenda: QT - Quimioterapia Fonte: Dados da pesquisa (2021)

Dentre as observações de impacto descritas, $82 \%(n=41)$ foram demonstradas de forma objetiva nos estudos, enquanto $18 \%(n=09)$ versou sobre o impacto em potencial, isto é, os resultados foram apenas citados nos estudos. Os impactos em potencial foram originários de quatro estudos com temas relacionados a fobia por agulha no contexto da quimioterapia, construção de bundle voltado ao extravasamento de antineoplásicos, desenvolvimento de diagnósticos de enfermagem voltados a mulheres com câncer de mama e, ainda, diagnósticos de enfermagem voltados a pacientes com câncer hematológico (Mendonça, Pereira, Magnago, Silva \& Martins, 2019; Melo et al., 2019; Naziazeno et al., 2020; Calegari, Cordeiro, Stacciarini \& Ferreira, 2018).

A promoção ao acesso à educação em saúde foi o impacto com maior frequência nos estudos, demonstrando o papel fundamental do Enfermeiro no processo de educação em saúde e como a sua atuação neste sentido, de fato, é capaz de ampliar o acesso do paciente às informações sobre seu contexto e tratamento.

Ademais, o alívio da carga de estresse ao paciente em Quimioterapia, encorajamento da adesão ao processo terapêutico e a promoção do conforto se mostraram como impactos gerados pela ação de Enfermeiros, corroborando com o entendimento de que a ação da enfermagem se faz diante de todos os aspectos individuais (biopsicosociocultural), proporcionando transformações significativas em todo o processo vivenciado pelo paciente em quimioterapia(Mendonça, Pereira, Magnago, Silva \& Martins, 2019; França et al., 2019; Silva, Signor, Pilati, Dalfollo \& Oliveira, 2019; Sueiro et al., 2019; Ferreira et al., 2017; Cunha, Vasconcelos, Silva \& Freitas, 2017; Cirilo, Silva, Fuly\& Moreira, 2016; Mesquita \& Silva, 2016).

Outrossim, no que se refere ao processo de quimioterapia em si, os impactos foram identificados em todos os momentos, desde intercorrências imediatas, ocorridas durante a infusão dos fármacos, até aos efeitos adversos a curto e longo prazo relacionados ao processo terapêutico. Em nove estudos foi demonstrado o amplo impacto da enfermagem na prevenção de intercorrências e de efeitos adversos relacionados à quimioterapia, promovendo a diminuição de suas ocorrências, e, ainda, manejo otimizado de intercorrências, evitando que se tornassem um dano ainda mais grave.

Desta forma, foi possível perceber queas ações do Enfermeiro voltadas aos pacientes oncológicos, no contexto quimioterápico, foram responsáveis por impactos positivos, assim como pela melhoria da qualidade da assistência. 


\section{Considerações Finais}

O estudo evidenciou o que a literatura científica aponta sobre as ações de Enfermeiros na assistência ao paciente oncológico em tratamento quimioterápico, no contexto brasileiro. Foi possível identificar as diversas áreas de atuação do Enfermeiro, com destaque para a área assistencial, gerenciamento e educação em saúde. Além disso, foi possível identificar que os enfermeiros se utilizam da elaboração de novas tecnologias para melhoria de processos em sua vivência cotidiana enquanto profissional. Sua atuação se mostrou necessária e com impactos positivos em todo o processo quimioterápico, sendo de grande relevância, inclusive, para prevenção de intercorrências.

O presente estudo possibilitou o entendimento do potencial de impacto e grande variabilidade de ações prestadas pelos Enfermeiros, demonstrando a importância de tal categoria profissional na assistência ao paciente em quimioterapia. Desta forma, evidencia-se a necessidade de maior valorização deste grupo de profissionais, por parte dos gestores e demais interessados, assim como o estabelecimento de estratégias de facilitação e de incentivo para o aperfeiçoamento e inovação de suas práticas, promovendo maior qualidade no manejo do paciente oncológico a curto, médio e longo prazo.

Como limitações do estudo evidencia-se a impossibilidade de utilização de instrumento validado para a avaliação dos impactos gerados pelas ações de enfermeiros. Além disto, foram considerados somente estudos com ações de enfermeiros, sendo recomendado demais investigações com a inclusão da categoria de técnicos em enfermagem a fim de extrapolar os achados.

Como trabalhos futuros, espera-se a realização de demais estudos voltados a atuação de Enfermeiros no manejo de pacientes oncológicos, contribuindo dessa forma, com novas estratégias a serem aplicadas no cotidiano assistencial, gerencial e educacional a fim de proporcionar melhoria da qualidade da assistência e das condições do paciente oncológico submetido à quimioterapia antineoplásica.

\section{Referências}

Bertolazzi, L. G. \& Perroca, M. G. (2020). Impacto das interrupções na duração das intervenções de enfermagem: Estudo em unidade de quimioterapia. Revista da Escola de Enfermagem da USP, 54. 10.1590/s1980-220x2018047503551.

Bryan, F., Ferlay, J., Soerjomataram, I., Siegel, R. L., Torre, L. A \& Jemal, A. (2018). Global cancer statistics 2018: GLOBOCAN estimates of incidence and mortality worldwide for 36 cancers in 185 countries. CA: A Cancer Journal for Clinicians,68(6),394-424.10.3322/caac.21492

Calegari, I.B., Cordeiro, A. L. P. C., Stacciarini, T. S. G. \& Ferreira, L.A. (2018). Diagnósticos de enfermagem em pacientes oncohematológicos submetidos a tratamento quimioterápico. Revista de Enfermagem e Atenção à Saúde,7(3), 102-115, DOI:10.18554/reas.v7i3.3116

Cirilo, J. D., Silva, M. M., Fuly, P. S. C. \& Moreira, M. C. (2016). A gerência do cuidado de enfermagem à mulher com câncer de mama em quimioterapia paliativa. Texto \& Contexto Enfermagem, 25(3). 10.1590/0104-07072016004130015

Coordenação de Aperfeiçoamento de Pessoas. (2019). Produção técnica - Relatório de Grupo de trabalho: Orientação CAPES, 1-81. (Trabalho original publicado em [2019]) <www.capes.gov.br/pt/relatorios-tecnicos-dav>

Cruz, F. O. A. M., Vieira, N. N. P., Manzi, N. M., Custódio, C. S., Ferreira, E. B. \& Reis, P. E. D. (2017). Implementação de manuais educativos na consulta de enfermagem: opinião dos pacientes submetidos à quimioterapia antineoplásica. Revista de Enfermagem UFPE OnLine, 11(5), 1757-1762. 10.5205/reuol.11077-98857-1-SM.1105201701

Cunha, F. F., Vasconcelos, E. V., Silva, S. E. D. \& Freitas, K. O. (2017). Representações de pacientes oncológicos sobre o tratamento de quimioterapia antineoplásica. Revista online de Pesquisa Cuidado é Fundamental, 9(3), 840-847. 10.9789/2175-5361.2017.v9i3.840-847

Eriksen, M. B. \& Frandsen T. F. (2018). The impact of patient, intervention, comparison, outcome (PICO) as a search strategy tool on literature search quality: a systematic review. Journal of the Medical Library 106(4), 420-431. DOI:10.5195/jmla.2018.345

Ferreira, E. B., Cruz, F.O.A.M., Jesus, C. A. C., Pinho, D. L. M., Kamada, I. \& Reis, P. E. D. (2017). Contato telefônico como estratégia para a promoção de conforto ao paciente submetido à quimioterapia. Revista de Enfermagem UFPE On Line, 11(5), 1936-1942, 10.5205/reuol.11077-98857-1-SM.1105201724

França, A. C., Rodrigues, A. B., Aguiar, M. I. F., Silva, R. A., Freitas, F. M. C. \& Melo, G. A. A. (2019). Telenfermagem para controle de náuseas e vômitos induzidos por quimioterapia: ensaio clínico randomizado. Texto \& Contexto Enfermagem, 28. 10.1590/1980-265X-TCE-2018-0404

Instituto Nacional de Câncer José Alencar Gomes da Silva [INCA]. (2020). Estimativa 2020: incidência de câncer no Brasil. Coordenação de Prevenção e Vigilância: Rio de Janeiro.

Jemal, A., Torre, L., Soerjomataram, I., Bray, F. (2019). The Cancer Atlas (3). American Cancer Society: Atlanta. 
Lopes, L. D., Rodrigues, A. B., Brasil, D. R. M., Moreira, M. M. C., Amaral, J. G. \& Oliveira, P. P. (2016). Prevenção e tratamento da mucosite em ambulatório de oncologia: uma construção coletiva. Texto \& Contexto Enfermagem, 25(1), 10.1590/0104-070720160002060014

Melnyk, B. M. \& Fineout-Overholt, E. (2011). Evidence-based practice in nursing \& healthcare: A guide to best practice ( $2^{\mathrm{a}}$ ed.). Philadelphia.

Melo, J. M. A., Oliveira, P. P., Rodrigues, A. B., Souza, R. S., Fonseca, D. F., Gontijo T. F., Silveira, E. A. A. (2020). Construção e avaliação de bundle frente ao extravasamento de antineoplásicos: estudo metodológico. Acta Paulista de Enfermagem, 33, 1-12. 10.37689/ acta-ape/2020AO0075

Mendes, K. D. S., Silveira, R. C. de C. P., \& Galvao, C. M.(2008) Revisão integrativa: método de pesquisa para a incorporação de evidências na saúde e na enfermagem. Texto \& Contexto Enfermagem, 17(4), 758-764, 10.1590/S0104-07072008000400018.

Mendonça, A. B., Pereira, E. R., Magnago, C., Silva, R. M. C. R. A. \& Martins, A. O. (2020). Processo de enfermagem para paciente com fobia de agulha: estudo de caso. Revista Brasileira de Enfermagem, 73(4). DOI:10.1590/0034-7167-2019-0095

Mesquita, M. E. R. \& Silva, R. P. (2016). Autocuidado e quimioterapia oral domiciliar: avaliação das práticas educativas dos enfermeiros sob a perspectiva de pacientes. Revista Brasileira de Cancerologia, 62(3), 237-245, DOI:10.32635/2176-9745.RBC.2016v62n3.165

Naziazeno, S. D. S., Melo, M. S., Andrade, J. S., Silva, J. R. S., Almeida, A. M. \& Gonçalves, L. L. C. (2020). Diagnósticos de enfermagem associados a qualidade de vida de mulheres com câncer de mama em quimioterapia. Revista online de Pesquisa Cuidado é Fundamental, 12, 629-635. 0.9789/21755361.rpcfo.v12.9083.

Oliveira, L. de A. M., Martins, C. R., Galvão, M. P. S. P., Júnior N. C. de A., Fontinele, A. V. C., Oliveira, C. P., Leite, M. F. F. da S., Araújo, M. P., Souza, J. M. \& Branco, R. V. F. C.(2019). Cuidados de enfermagem realizados no tratamento quimioterápico do câncer do colo do útero: uma revisão integrativa. Brazilian Journal of Surgery and Clinical Research, 26(2),70-74.

Perdigão, M. M. M., Rodrigues, A. B., Magalhães, T. L., Freitas, F. M. C., Bravo, L. G. \& Oliveira, P. P. (2019). Tecnologia educativa para manejo da fadiga relacionada à quimioterapia antineoplásica. Revista Brasileira de Enfermagem, 72(6), 1596-1602. 10.1590/0034-7167-2018-0505

Pinto, A., Guimarães, V. \& Lanza, L. (2017). Espiritualidade e o enfrentamento de pacientes submetidos à quimioterapia. Revista da Faculdade de Ciências Médicas de Sorocaba, 19(2), 81-85. DOI:10.23925/1984-4840.2017v19i2a7

Silva, L. C. A., Signor, A. C., Pilati, A. C. L. \& Dalfollo, B. R. (2019). Abordagem Educativa ao Paciente Oncológico: Estratégias para Orientação acerca do Tratamento Quimioterápico. Revista Brasileira de Cancerologia, 65(1), 0.32635/2176-9745.RBC.2019v65n1.305

Silva, S. S. F., Monteiro, J. de A. S., Silva, L. R., Francisco, M. R., Brasil, Y. Z. \& Paula, D. G. (2020). Produções tecnológicas desenvolvidas por Enfermagem: revisão sistemática. Research, Society and Development, 9(8). 10.33448/rsd-v9i8.5721

Souza, N. R., Bushatsky, M., Figueiredo, E. G., Melo, J. T. S., Freire, D. A. \& Santos, I. C. R. V. (2016). Emergência oncológica: atuação dos enfermeiros no extravasamento de drogas quimioterápicas antineoplásicas. Escola Anna Nery, 21(1), 10.5935/1414-8145.20170009

Sueiro, I.M, Góes, F. G. B., Martins, A. S., Moraes, J. R. M. M., Silva, L. J. \& Silva, L. F. (2019). Fatores intervenientes para o cuidado de enfermagem na alimentação da criança hospitalizada em quimioterapia. Revista de Enfermagem da UFSM, 9(25), 1-19, DOI:10.5902/2179769231138

Sueiro, I. M., Góes, F. G. B., Silva, L. F. \& Moraes, J. R. M. M. (2019). Cuidados de Enfermagem da Alimentação de Crianças em Quimioterapia: Contribuições de Collière. Revista online de Pesquisa Cuidado é Fundamental, 11, 351-357, 10.9789/2175-531.2019.v11i2.351-357

Tolentino, G. S., Bettencourt, A. R. C. \& Fonseca, S. M. (2019). Construção e validação de instrumento para consulta de enfermagem em quimioterapia ambulatorial. Revista Brasileira de Enfermagem, 72(2), 409-417, 10.1590/0034-7167-2018-0031 